\title{
An efficient and eco-friendly synthesis of 2-amino-1,3-selenazoles in an ionic liquid/water system under ambient conditions
}

\author{
Taterao M. Potewar, Sachin A. Ingale, and Kumar V. Srinivasan* \\ Division of Organic Chemistry, National Chemical Laboratory, \\ Dr. Homi Bhabha Road, Pune-411 008, India \\ E-mail: kumarv.srinivasan@gmail.com
}

\begin{abstract}
An efficient and eco-friendly synthesis of 2-amino-1,3-selenazoles in ionic liquid/water solvent system is described. The paper describes synthesis of 2-amino-1,3-selenazoles by condensation of various phenacyl bromide with selenourea under ambient conditions in short reaction times. This protocol has notable advantages such as the absence of any added catalyst, simple operation and excellent yields of products and recyclability of the ionic liquid.
\end{abstract}

Keywords: 2-Amino-1,3-selenazoles, selenoureas, substituted phenacyl bromide, ionic liquid/water system, ambient conditions, heterocycles

\section{Introduction}

The synthesis of selenium containing heterocycles has been extensively studied, not only because of strong interest in these compounds as synthetic tools, ${ }^{1}$ but also as a result of their biological activities. ${ }^{2}$ 1,3-Selenazoles are of pharmacological relevance, due to their antibiotic and cancerostatic activity. ${ }^{3}$ A prominent example is the antibiotically active C-glycosyl selenazole, selenafurin. ${ }^{3 a}$ Recently, it was reported that 1,3 -selenazole possess strong inhibitory activity against inducible nitric oxide synthase. ${ }^{4}$ 2-Amino-1,3-selenazole are also good superoxide anion-scavengers. ${ }^{5}$ Moreover, 2-dialkylamino-1,3-selenazole ${ }^{6 a}$ has become of interest as a starting material for preparing dyes. ${ }^{6 \mathrm{~b}-\mathrm{c}}$

In view of the importance of selenazole and its derivatives, several methods for the synthesis of selenazoles were reported. The most widely used method is by the application of Hantzsch procedure. ${ }^{7,8}$ Most of the methods available for the synthesis of selenazole had drawbacks such as use of polar and anhydrous organic solvents at higher temperature, ${ }^{8}$ basic reaction conditions such as use of triethylamine/ammonia, ${ }^{8 \mathrm{f}}$ use of catalyst such as $\beta$-cyclodextrin, ${ }^{8 \mathrm{a}}$ longer reaction times and low yields in the presence of water. ${ }^{9}$ Furthermore, selenoureas are air and light sensitive, so there is a need to develop a process under mild conditions such as ambient 
temperature at enhanced rate of reaction to prevent the unstable selenoureas triggering unwanted side reactions thereby reducing their air and light sensitivity thereby improving the selectivity to the required 2-aminoselenazoles.

Thus the development of a simple, convenient and environment friendly approach for the synthesis of 1,3-selenazole is still needed. Organic reactions in aqueous media have attracted much attention recently not only because water is the most abundant, environmentally benign, and cheapest solvent but also because unique selectivity and reactivity can be expected from this solvent. ${ }^{10}$ Among a variety of possible green solvent alternatives for catalytic reactions, roomtemperature ionic liquid (RTIL) reaction media continue to be an area of increasing research activity. Moreover, they have shown great promise as attractive alternatives to conventional solvents. They possess the unique advantages of high thermal stability, negligible vapour pressure, immiscibility with a number of organic solvents and recyclability. ${ }^{11}$ These advantages become even more attractive if such reactions can be conducted using ionic liquids in aqueous media. $^{12}$

In continuation to our ongoing interest in the development of green organic synthetic processes for the preparation of biologically active heterocycles by the use of ionic liquid, ${ }^{13}$ we herein report for the first time a simple, efficient and eco-friendly process for the synthesis of substituted 2-amino-1,3-selenazoles in ionic liquid/water mixture as an efficient solvent system at ambient temperature in significantly enhanced reaction rates.

\section{Results and Discussion}

When $p$-chlorophenacyl bromide 1d was treated with selenourea 2 in 1-n-butylimidazolium tetrafluoroborate, $\left([\mathrm{Hbim}] \mathrm{BF}_{4}\right) / \mathrm{H}_{2} \mathrm{O}$ mixture $(1: 1)$ at ambient temperature, it afforded $4-(p$ chlorophenyl)-2-amino-1,3-selenazole 3d in 94\% yield in 10 min (Scheme 1).

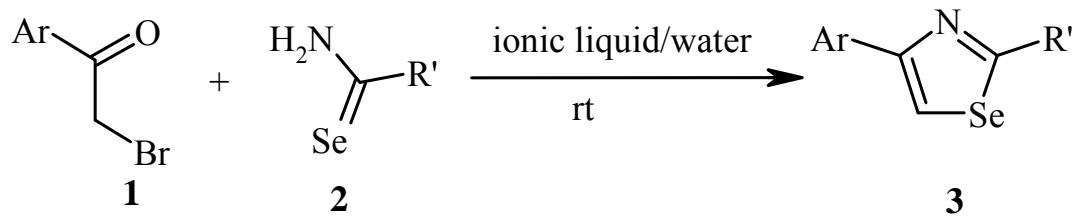

\section{Scheme 1}

For the optimization of the reaction conditions, we chose the cyclocondensation reaction between $p$-chlorophenacyl bromide and selenourea as a model reaction. Initially, we carried out the reaction between $p$-chlorophenacyl bromide and selenourea in water at room temperature, where it furnished the desired product in low yield (58\%) even after long reaction period $(6 \mathrm{~h})$ (Table 1, Entry 8). Next, when we carried out the reaction in the IL, 1,3-di-n-butylimidazolium bromide ([Bbim]Br), it furnished the product in $91 \%$ in 35 min (Table 1, Entry 1). However, a 
similar reaction in more Brønsted acidic IL, 1- $n$-butylimidazolium tetrafluoroborate $\left([\mathrm{Hbim}] \mathrm{BF}_{4}\right)$ furnished the product in 94\% yield in 15 min (Table 1, Entry 2). Interestingly, it was observed that addition of water to ionic liquid, $[\mathrm{Hbim}] \mathrm{BF}_{4}$ in $1: 1$ ratio enhanced the rate of cyclocondensation reaction as reaction time was reduced from $15 \mathrm{~min}$ to $10 \mathrm{~min}$ while in the case of [Bbim] $\mathrm{Br}$, the reaction time was reduced from $35 \mathrm{~min}$ to $25 \mathrm{~min}$ on addition of water to [Bbim] $\mathrm{Br}$ in 1:1 ratio. Thus, the reaction in $[\mathrm{Bbim}] \mathrm{Br} / \mathrm{H}_{2} \mathrm{O}$ mixture (1:1) was found to be relatively sluggish as compared to the $[\mathrm{Hbim}] \mathrm{BF}_{4} / \mathrm{H}_{2} \mathrm{O}$ mixture. It may be pointed out that the ionic liquid $[\mathrm{Hbim}] \mathrm{BF}_{4}$ and $[\mathrm{Bbim}] \mathrm{Br}$ were selected for this transformation because of their excellent miscibility with water. The role of IL may be postulated in terms of Lewis/Brønsted acidity, thereby promoting the reaction. The addition of water to IL may presumably play an important role in the process due to the good water solubility of the selenoureas.

Table 1. Optimization of solvent system for the synthesis of $\mathbf{3 d}$

\begin{tabular}{|c|c|c|c|}
\hline Entry & Solvent & $\begin{array}{l}\text { Time } \\
\text { (min) }\end{array}$ & $\begin{array}{l}\text { Yield } \\
(\%)^{\mathrm{a}}\end{array}$ \\
\hline 1 & [Bbim]Br & 35 & 91 \\
\hline 2 & {$\left[\mathrm{Hbim} \mathrm{BF}_{4}\right.$} & 15 & 94 \\
\hline 3 & {$[\mathrm{Hbim}] \mathrm{BF}_{4}+\mathrm{H}_{2} \mathrm{O}(1: 1)$} & 10 & 94 \\
\hline 4 & {$[\mathrm{Hbim}] \mathrm{BF}_{4}+\mathrm{H}_{2} \mathrm{O}(2: 1)$} & 10 & 94 \\
\hline 5 & {$[\mathrm{Hbim}] \mathrm{BF}_{4}+\mathrm{H}_{2} \mathrm{O}(1: 2)$} & 30 & 89 \\
\hline 6 & {$[\mathrm{Bbim}] \mathrm{Br}+\mathrm{H}_{2} \mathrm{O}(1: 1)$} & 25 & 90 \\
\hline 7 & $\mathrm{H}_{2} \mathrm{O}+[\mathrm{Hbim}] \mathrm{BF}_{4}^{\mathrm{b}}$ & 60 & 87 \\
\hline 8 & $\mathrm{H}_{2} \mathrm{O}$ & $120^{\mathrm{c}}$ & 58 \\
\hline
\end{tabular}

${ }^{a}$ Isolated yield after column chromatography.

${ }^{\mathrm{b}} \mathrm{IL},[\mathrm{Hbim}] \mathrm{BF}_{4}$ in catalytic amount.

${ }^{\mathrm{c}}$ No further progress upto $6 \mathrm{~h}$.

For optimization of solvent system, different ratios of water and IL were investigated and the optimum results were obtained by using IL/water in 1:1 ratio (Table 1, entry 3). All further reactions were done using $[\mathrm{Hbim}] \mathrm{BF}_{4} / \mathrm{H}_{2} \mathrm{O}$ system $(1: 1)$ as the reaction medium. Using these optimal reaction conditions, we next examined the scope and generality of this process. Several 2-amino- and $N, N$-dimethylamino-1,3-selenazoles 3a-m were prepared from a variety of substituted phenacyl bromides and selenoureas and results are summarized in Table 2. It was observed that under these conditions, a wide range of phenacyl bromides having electronwithdrawing as well as electron-donating groups such as nitro, chloro, fluoro, methyl and phenyl easily underwent cyclocondensation with selenourea as well as with $N, N$-dimethylselenourea to afford substituted 4-aryl-2-amino-1,3-selenazole in short reaction times in excellent isolated yields. Similarly, $\alpha$-bromo-2-acetonaphthone smoothly reacted with selenourea and $N, N$ - 
dimethylselenourea to afford the corresponding products in excellent yields (Table 2, Entry 7,13).

Table 2. Synthesis of 2-amino-1,3-selenazoles 3a-m in IL/water system

\begin{tabular}{|c|c|c|c|c|c|c|}
\hline Entry & $\mathrm{Ar}$ & $\mathrm{R}^{\prime}$ & Product 3 & $\begin{array}{l}\text { Time } \\
(\mathrm{min})\end{array}$ & Yield $(\%)^{\mathrm{a}}$ & $\mathrm{Mp}\left({ }^{\circ} \mathrm{C}\right)$ \\
\hline 1 & $\mathrm{C}_{6} \mathrm{H}_{5}$ & $\mathrm{NH}_{2}$ & 3a & 10 & 91 & $\begin{array}{c}131-132 \\
(132)^{8 a}\end{array}$ \\
\hline 2 & $4-\mathrm{MeC}_{6} \mathrm{H}_{4}$ & $\mathrm{NH}_{2}$ & $3 \mathbf{b}$ & 10 & 93 & $\begin{array}{c}167-168 \\
(167)^{8 a}\end{array}$ \\
\hline 3 & $4-\mathrm{FC}_{6} \mathrm{H}_{4}$ & $\mathrm{NH}_{2}$ & $3 c$ & 15 & 94 & $119-120$ \\
\hline 4 & $4-\mathrm{ClC}_{6} \mathrm{H}_{4}$ & $\mathrm{NH}_{2}$ & 3d & 10 & 94 & $\begin{array}{c}155-156 \\
(157)^{8 a}\end{array}$ \\
\hline 5 & $3-\left(\mathrm{O}_{2} \mathrm{~N}\right) \mathrm{C}_{6} \mathrm{H}_{4}$ & $\mathrm{NH}_{2}$ & $3 e$ & 15 & 97 & 196-197 \\
\hline 6 & $4-\mathrm{C}_{6} \mathrm{H}_{5} \mathrm{C}_{6} \mathrm{H}_{4}$ & $\mathrm{NH}_{2}$ & $3 f$ & 20 & 96 & 138-139 \\
\hline 7 & $\beta-\mathrm{C}_{10} \mathrm{H}_{7}$ & $\mathrm{NH}_{2}$ & $3 g$ & 10 & 95 & $145-146$ \\
\hline 8 & $\mathrm{C}_{6} \mathrm{H}_{5}$ & $\mathrm{NMe}_{2}$ & $3 \mathbf{h}^{\mathrm{b}}$ & 10 & 94 & 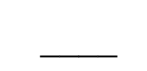 \\
\hline 9 & 4- $\mathrm{MeC}_{6} \mathrm{H}_{4}$ & $\mathrm{NMe}_{2}$ & $3 \mathbf{i}$ & 10 & 95 & $49-50$ \\
\hline 10 & $4-\mathrm{FC}_{6} \mathrm{H}_{4}$ & $\mathrm{NMe}_{2}$ & $3 \mathbf{j}^{\mathrm{b}}$ & 10 & 96 & 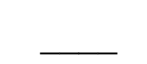 \\
\hline 11 & $3-\left(\mathrm{O}_{2} \mathrm{~N}\right) \mathrm{C}_{6} \mathrm{H}_{4}$ & $\mathrm{NMe}_{2}$ & $3 \mathbf{k}$ & 15 & 97 & $104-105$ \\
\hline 12 & $4-\mathrm{C}_{6} \mathrm{H}_{5} \mathrm{C}_{6} \mathrm{H}_{4}$ & $\mathrm{NMe}_{2}$ & 31 & 10 & 96 & $127-128$ \\
\hline 13 & $\beta-\mathrm{C}_{10} \mathrm{H}_{7}$ & $\mathrm{NMe}_{2}$ & $3 m$ & 10 & 97 & $123-124$ \\
\hline
\end{tabular}

${ }^{\mathrm{a}}$ Isolated yield after column chromatography.

bliquid.

It can be observed that almost all reactions were complete in just 10-20 min at ambient temperature. Selenoureas are air and light sensitive, so it becomes most necessary to develop a 
process which would be faster at mild conditions to avoid the unstable selenoureas triggering unwanted side reactions thereby reducing their air and light sensitivity thereby improving the selectivity to the required 2-aminoselenazoles. To the best of our knowledge, this is the first synthesis of 2-amino-1,3-selenazole which was carried out at ambient temperature at enhanced rate without any volatile, polar organic solvent and any added catalyst affording the products in excellent yields (91-97\%). All the products were characterized by their melting point, IR, elemental analyses, ${ }^{1} \mathrm{H}$ and ${ }^{13} \mathrm{C}$ NMR spectroscopy. For known compound, the values were in good agreement to those reported in literature.

The experimental procedure is very simple. A mixture of phenacyl bromide $\mathbf{1}$ and selenourea 2 was stirred in $[\mathrm{Hbim}] \mathrm{BF}_{4} / \mathrm{H}_{2} \mathrm{O}(1: 1)$ system for $10-20 \mathrm{~min}$ at ambient temperature. The vigorous stirring is necessary for the success of the reaction. After completion of the reaction, as indicated by the disappearance of the spot corresponding to phenacyl bromide on TLC, the reaction mixture was poured into water $(10 \mathrm{~mL})$ and product was extracted using ethyl acetate. The aqueous layer consisting of the IL was dried in vacuo $\left(80{ }^{\circ} \mathrm{C}\right.$ at $\left.10 \mathrm{mmHg}\right)$ to remove water, leaving behind the IL, $[\mathrm{Hbim}] \mathrm{BF}_{4}$ which was reused and recycled three times for a typical reaction of $p$-chlorophenacyl bromide and selenourea without any loss in yield. For this, recovered IL was mixed with water in 1:1 ratio and used as the solvent system.

\section{Conclusions}

In conclusion, we have demonstrated that a highly efficient and eco-friendly synthesis of 4-aryl2-amino-1,3-selenazole has been achieved using [ $\mathrm{Hbim}] \mathrm{BF}_{4} / \mathrm{H}_{2} \mathrm{O}$ solvent system. The current method presents a practicable synthetic process for 2-amino-1,3-selenazole because of the following advantages: (1) use of water and ionic liquid as environmentally benign reaction media, (2) very high yield (91-97\%) and short reaction time (10-20 min), (3) ambient temperature, (4) absence of any added catalyst and organic solvent as reaction medium and (5) recyclability of the ionic liquid.

\section{Experimental Section}

General Procedures. ${ }^{1} \mathrm{H}$ NMR and ${ }^{13} \mathrm{C}$ NMR spectra were recorded on a Bruker AV-200 spectrometer in $\mathrm{CDCl}_{3}$ and $\mathrm{DMSO}-\mathrm{d}_{6}$ using TMS as internal standard. Infrared spectra were recorded with ATI MATT-SON RS-1 FTIR spectrometer using KBr pellets. Elemental analyses were obtained using a flash EA 1112 thermofinnigan instrument and were carried out at the National Chemical Laboratory, Pune, India. Melting points were recorded in open capillary on Buchi melting Point B-540 apparatus. All solvents and chemicals were of research grade and were used as obtained from Merck and Lancaster. Column chromatography was performed using silica gel (60-120 mesh size). 


\section{General procedures for the synthesis of 2-aminoselenazoles (3a-m)}

A mixture of phenacyl bromide $1(1 \mathrm{mmol})$ and selenourea $2(1.1 \mathrm{mmol})$ in $\left([\mathrm{Hbim}] \mathrm{BF} / \mathrm{water}_{4}\right.$ $(1: 1,3 \mathrm{~mL})$ system was vigorously stirred at ambient temperature for an appropriate time (Table $2)$. After completion of the reaction as indicated by TLC, the reaction mixture was poured into water $(10 \mathrm{~mL})$ and the product was extracted using ethyl acetate $(3 \mathrm{X} 10 \mathrm{~mL})$. The combined ethyl acetate extracts were concentrated in vacuo. The resulting crude product was directly charged onto a small silica gel column and eluted with a mixture of ethyl acetate/petroleum ether to afford pure 2-aminoselenazole 3.

4-(4-Fluorophenyl)-1,3-selenazol-2-amine (3c). Brown solid; mp 119-120 ${ }^{\circ} \mathrm{C}$; IR ( $\left.\mathrm{KBr}, \mathrm{cm}^{-1}\right)$ 3454, 3279, 1696, 1459, 1377, 1321, 1208, 771, 728; ${ }^{1} \mathrm{H}$ NMR $\left(\mathrm{CDCl}_{3}, 200 \mathrm{MHz}\right) \delta 5.36$ (bs, $\left.2 \mathrm{H}, \mathrm{NH}_{2}\right), 6.99-7.08(\mathrm{~m}, 2 \mathrm{H}, \mathrm{ArH}), 7.20(\mathrm{~s}, 1 \mathrm{H}$, selenazole $\mathrm{H}), 7.70-7.77(\mathrm{~m}, 2 \mathrm{H}, \mathrm{ArH}) ;{ }^{13} \mathrm{C}$ $\mathrm{NMR}\left(\mathrm{CDCl}_{3}, 50 \mathrm{MHz}\right) \delta 106.7,115.1,115.5,127.8,128.0,131.7,131.7,150.8,159.7,164.6$, 169.3; Anal Calcd $\mathrm{C}_{9} \mathrm{H}_{7} \mathrm{FN}_{2} \mathrm{Se}: \mathrm{C}, 44.83 ; \mathrm{H}, 2.93 ; \mathrm{N}, 11.62 \%$. Found: $\mathrm{C}, 44.69 ; \mathrm{H}, 2.84 ; \mathrm{N}$, $11.77 \%$.

4-(3-Nitrophenyl)-1,3-selenazol-2-amine (3e). Dark yellow solid; mp 196-197 ${ }^{\circ} \mathrm{C}$; IR $(\mathrm{KBr}$, $\left.\mathrm{cm}^{-1}\right)$ 3443, 1633, 1463, 1455, 1155, 771, 722; ${ }^{1} \mathrm{H}$ NMR $\left(\mathrm{CDCl}_{3} / \mathrm{DMSO}_{6}, 200 \mathrm{MHz}\right) \delta 6.49$ (bs, 2H, $\mathrm{NH}_{2}$ ), 7.0 (s, 1H, selenazole H), 7.08 (t, J $\left.7.98 \mathrm{~Hz}, 1 \mathrm{H}, \mathrm{ArH}\right), 7.59-7.69$ (m, 2H, ArH), $8.22(\mathrm{t}, J 1.98 \mathrm{~Hz}, 1 \mathrm{H}, \mathrm{ArH}) ;{ }^{13} \mathrm{C} \mathrm{NMR}\left(\mathrm{CDCl}_{3} / \mathrm{DMSO}_{6}, 50 \mathrm{MHz}\right) \delta 107.0,119.7,120.1$, 128.2, 130.5, 136.2, 147.2, 147.6, 169.0; Anal Calcd $\mathrm{C}_{9} \mathrm{H}_{7} \mathrm{~N}_{3} \mathrm{O}_{2} \mathrm{Se}: \mathrm{C}, 40.32 ; \mathrm{H}, 2.63 ; \mathrm{N}, 15.67 \%$. Found: C, 40.51; H, 2.74; N, 15.53\%.

4-(4-Phenylphenyl)-1,3-selenazol-2-amine (3f). Brown solid; mp 138-139 ${ }^{\circ} \mathrm{C}$; IR $\left(\mathrm{KBr}, \mathrm{cm}^{-1}\right)$ 3412, 1538, 1462, 1376, 769, 733; ${ }^{1} \mathrm{H}$ NMR $\left(\mathrm{CDCl}_{3}, 200 \mathrm{MHz}\right) \delta 5.30$ (bs, 2H, NH$) 7.34(\mathrm{~s}, 1 \mathrm{H}$, selenazole H), 7.36-7.48 (m, 3H, ArH), 7.58-7.65 (m, 4H, ArH), 7.82-7.87 (m, 2H); ${ }^{13} \mathrm{C} \mathrm{NMR}$ $\left(\mathrm{CDCl}_{3} / \mathrm{DMSO}_{6}, 50 \mathrm{MHz}\right) \delta 104.0,124.5,124.7,125.3,126.9,132.9,136.7,138.0,148.5$, 167.5; Anal Calcd $\mathrm{C}_{15} \mathrm{H}_{12} \mathrm{~N}_{2}$ Se: C, 60.21; H, 4.04; N, 9.36\%. Found: C, 60.37; H, 4.12; N, $9.23 \%$.

4-(Naphthalen-2-yl)-1,3-selenazol-2-amine (3g). Brown solid; mp 145-146 ${ }^{\circ} \mathrm{C}$; IR ( $\mathrm{KBr}, \mathrm{cm}^{-1}$ ) 3415, 1595, 1519, 1462, 1377, 770, 749; ${ }^{1} \mathrm{H}$ NMR $\left(\mathrm{CDCl}_{3}, 200 \mathrm{MHz}\right) \delta 5.47$ (bs, 2H, NH$), 7.41$ (s, $1 \mathrm{H}$, selenazole H), 7.43-7.53 (m, 2H, ArH), 7.48-7.89 (m, 4H, ArH), $8.30(\mathrm{~s}, 1 \mathrm{H}, \mathrm{ArH}) ;{ }^{13} \mathrm{C}$ NMR $\left(\mathrm{CDCl}_{3} / \mathrm{DMSO}_{6}, 50 \mathrm{MHz}\right) \delta 105.5,123.2,123.8,124.6,125.1,126.4,126.8,127.0$, 131.4, 132.0, 132.4, 149.9, 168.8; Anal Calcd $\mathrm{C}_{13} \mathrm{H}_{10} \mathrm{~N}_{2} \mathrm{Se}$ : C, 57.15; H, 3.69; N, 10.25\%. Found: C, 57.04; H, 3.82; N, 10.37\%.

$\boldsymbol{N}, \boldsymbol{N}$-Dimethyl-4-phenyl-1,3-selenazol-2-amine (3h). Viscous liquid; IR ( $\left.\mathrm{KBr}, \mathrm{cm}^{-1}\right) 2921$, 2871, 1566, 1479, 773; ${ }^{1} \mathrm{H}$ NMR $\left(\mathrm{CDCl}_{3}, 200 \mathrm{MHz}\right) \delta 3.26\left(\mathrm{~s}, 6 \mathrm{H}, \mathrm{CH}_{3}\right), 7.38(\mathrm{~s}, 1 \mathrm{H}$, selenzole $\mathrm{H}), 7.40-7.52$ (m, 3H, ArH), 7.96-8.01 (m, 2H, ArH); ${ }^{13} \mathrm{C} \mathrm{NMR}\left(\mathrm{CDCl}_{3}, 50 \mathrm{MHz}\right) \delta 40.9,105.0$, 126.3, 127.2, 128.3, 136.0, 153.0, 172.1; Anal Calcd $\mathrm{C}_{11} \mathrm{H}_{12} \mathrm{~N}_{2} \mathrm{Se}$ : C, 52.60; H, 4.82; N, 11.15\%. Found: C, 52.47; H, 4.94; N, 11.03\%.

$\boldsymbol{N}, \boldsymbol{N}$-Dimethyl-4-p-tolyl-1,3-selenazol-2-amine (3i). Dark brown solid; mp 49-50 ${ }^{\circ} \mathrm{C}$; IR ( $\mathrm{KBr}$, $\left.\mathrm{cm}^{-1}\right) 3018,2922,2872,1563,1490,1554,1425,1322,1215,758 ;{ }^{1} \mathrm{H} \mathrm{NMR}\left(\mathrm{CDCl}_{3}, 200 \mathrm{MHz}\right)$ $\delta 2.35\left(\mathrm{~s}, 3 \mathrm{H}, \mathrm{CH}_{3}\right), 3.15\left(\mathrm{~s}, 6 \mathrm{H}, \mathrm{CH}_{3}\right), 7.15-7.19(\mathrm{~d}, J 7.96 \mathrm{~Hz}, 2 \mathrm{H}, \mathrm{ArH}), 7.21(\mathrm{~s}, 1 \mathrm{H}$, selenazole 
$\mathrm{H}), 7.74-7.78(\mathrm{~d}, J 7.96 \mathrm{~Hz}, 2 \mathrm{H}, \mathrm{ArH}) ;{ }^{13} \mathrm{C} \mathrm{NMR}\left(\mathrm{CDCl}_{3}, 50 \mathrm{MHz}\right) \delta 21.2,40.9,104.1,126.2$, 129.0, 133.3, 136.9, 135.1, 172.1; Anal Calcd $\mathrm{C}_{12} \mathrm{H}_{14} \mathrm{~N}_{2} \mathrm{Se}$ : C, 54.35; H, 5.32; N, 10.56\%. Found: C, $54.53 ; \mathrm{H}, 5.21 ; \mathrm{N}, 10.68 \%$.

$N, N$-Dimethyl-4-(4-fluorophenyl)-1,3-selenazol-2-amine (3j). Viscous liquid; IR ( $\left.\mathrm{KBr}, \mathrm{cm}^{-1}\right)$ 2921, 2872, 1572, 1489, 843, 756, 728; ${ }^{1} \mathrm{H}$ NMR $\left(\mathrm{CDCl}_{3}, 200 \mathrm{MHz}\right) \delta 3.14\left(\mathrm{~s}, 6 \mathrm{H}, \mathrm{CH}_{3}\right), 6.99-$ $7.08(\mathrm{~m}, 2 \mathrm{H}, \mathrm{ArH}), 7.17(\mathrm{~s}, 1 \mathrm{H}$, selenazole $\mathrm{H}), 7.79-7.86(\mathrm{~m}, 2 \mathrm{H}, \mathrm{ArH}) ;{ }^{13} \mathrm{C} \mathrm{NMR}\left(\mathrm{CDCl}_{3}, 50\right.$ MHz) $\delta 41.0,104.4,114.9,115.3,127.9,128.0,132.3,132.3,152.0,159.6,164.2,172.2$; Anal Calcd $\mathrm{C}_{11} \mathrm{H}_{11} \mathrm{FN}_{2} \mathrm{Se}: \mathrm{C}, 49.08 ; \mathrm{H}, 4.12 ; \mathrm{N}, 10.41 \%$. Found: $\mathrm{C}, 49.17 ; \mathrm{H}, 4.23 ; \mathrm{N}, 10.58 \%$.

$N, N$-Dimethyl-4-(3-nitrophenyl)-1,3-selenazol-2-amine (3k). Dark yellow solid; mp 104-105 ${ }^{\circ} \mathrm{C}$; IR $\left(\mathrm{KBr}, \mathrm{cm}^{-1}\right)$ 2924, 1566, 1529, 1348, 1048, 770, 697; ${ }^{1} \mathrm{H}$ NMR $\left(\mathrm{CDCl}_{3}, 200 \mathrm{MHz}\right) \delta 3.17$ (s, 6H, $\left.\mathrm{CH}_{3}\right), 7.41(\mathrm{~s}, 1 \mathrm{H}$, selenazole H), $7.51(\mathrm{t}, J 7.89 \mathrm{~Hz}, 1 \mathrm{H}, \mathrm{ArH}), 8.07-8.19(\mathrm{~m}, 2 \mathrm{H}, \mathrm{ArH})$, $8.69(\mathrm{t}, J 1.96 \mathrm{~Hz}, 1 \mathrm{H}, \mathrm{ArH}) ;{ }^{13} \mathrm{C} \mathrm{NMR}\left(\mathrm{CDCl}_{3}, 50 \mathrm{MHz}\right) \delta 41.0,107.2,121.2,121.7,129.2$, 132.0, 137.5, 148.5, 150.6, 172.4; Anal Calcd $\mathrm{C}_{11} \mathrm{H}_{11} \mathrm{~N}_{3} \mathrm{O}_{2} \mathrm{Se}: \mathrm{C}, 44.61 ; \mathrm{H}, 3.74 ; \mathrm{N}, 14.19$. Found: C, 44.48; H, 3.91; N, 14.33\%.

$\boldsymbol{N}, \boldsymbol{N}$-Dimethyl-4-(4-phenylphenyl)-1,3-selenazol-2-amine (3l). Brown solid; mp 127-126 ${ }^{\circ} \mathrm{C}$; IR $\left(\mathrm{KBr}, \mathrm{cm}^{-1}\right)$ 2921, 2872, 1574, 1477, 848, 730; ${ }^{1} \mathrm{H}$ NMR $\left(\mathrm{CDCl}_{3}, 200 \mathrm{MHz}\right) \delta 3.17(\mathrm{~s}, 6 \mathrm{H}$, $\left.\mathrm{CH}_{3}\right), 7.31(\mathrm{~s}, 1 \mathrm{H}$, selenazole $\mathrm{H}), 7.33-7.48(\mathrm{~m}, 3 \mathrm{H}, \mathrm{ArH})$, 7.58-7.66 (m, 4H, $\left.\mathrm{ArH}\right), 7.92-7.96$ (dd, $J 8.41$ and $1.76 \mathrm{~Hz}, 2 \mathrm{H}, \mathrm{ArH}) ;{ }^{13} \mathrm{C} \mathrm{NMR}\left(\mathrm{CDCl}_{3}, 50 \mathrm{MHz}\right) \delta 41.0,105.1,126.7,126.8$, 127.0, 128.6, 135.1, 139.8, 140.8, 152.7, 172.1; Anal Calcd $\mathrm{C}_{17} \mathrm{H}_{16} \mathrm{~N}_{2} \mathrm{Se}: \mathrm{C}, 62.39 ; \mathrm{H}, 4.93 ; \mathrm{N}$, $8.56 \%$. Found: C, $62.28 ; \mathrm{H}, 4.81 ; \mathrm{N}, 8.67 \%$.

$N$, $N$-Dimethyl-4-(naphthalen-2-yl)-1,3-selenazol-2-amine (3m). Brown solid; mp 123-124 ${ }^{\circ} \mathrm{C}$; IR $\left(\mathrm{KBr}, \mathrm{cm}^{-1}\right) 3018,2924,1560,770 ;{ }^{1} \mathrm{H} \mathrm{NMR}\left(\mathrm{CDCl}_{3}, 200 \mathrm{MHz}\right) \delta 3.20\left(\mathrm{~s}, 6 \mathrm{H}, \mathrm{CH}_{3}\right), 7.40(\mathrm{~s}$, $1 \mathrm{H}$, selenazole H), 7.42-7.49 (m, 2H, ArH), 7.78-7.96 (m, 4H, ArH), $8.40(\mathrm{~s}, 1 \mathrm{H}, \mathrm{ArH}) ;{ }^{13} \mathrm{C}$ NMR $\left(\mathrm{CDCl}_{3}, 50 \mathrm{MHz}\right) \delta 41.0,104.4,114.9,115.3,127.9,128.0,132.3,152.0,159.6,164.5$, 172.2; Anal Calcd $\mathrm{C}_{15} \mathrm{H}_{14} \mathrm{~N}_{2} \mathrm{Se}$ : C, 59.81; H, 4.68; N, 9.30\%. Found: C, 59.89; H, 4.77; N, $9.37 \%$.

\section{Acknowledgements}

T.M.P. thanks the CSIR, New Delhi, for the award of Senior Research Fellowship.

\section{References}

1. (a) Casar, Z.; Majcen-Le Marechal, A.; Lorcy, D. New J. Chem. 2003, 27, 1622. (b) Koketsu, M.; Ishihara, H. Curr. Org. Chem. 2003, 7, 175. (c) Duddeck, H.; Bradenahl, R.; Stefaniak, L.; Jazwinski, J.; Kamienski, B. Magn. Reson. Chem. 2001, 39, 709. (d) Archer, S.; McGarry, R. J. Heterocycl. Chem. 1982, 19, 1245. 
2. (a) Koketsu, M.; Choi, S. Y.; Ishihara, H.; Lim, B. O.; Kim, H.; Kim, S. Y. Chem. Pharm. Bull. 2002, 50, 1594. (b) Li, H.; Hallows, W. H.; Punzi, J. S.; Marquez, V. E.; Carrell, H. L.; Pankiewicz, K. W.; Watanabe, K. A.; Goldstein, B. M. Biochemistry 1994, 33, 23. (c) Burling, F. T.; Goldstein, B. M. J. Am. Chem. Soc. 1992, 114, 2313.

3. (a) Goldstein, B. M.; Kennedy, S. D.; Hennen, W. J. J. Am. Chem. Soc. 1990, 112, 8265 and references cited therein. (b) Shafiee, A.; Khashayarmanesh, Z.; Kamal, F. J. Sci., Islamic Repub. Iran 1990, 1, 11. (c) Shafiee, A.; Shafaati, A.; Khamench, B. H. J. Heterocycl. Chem. 1989, 26, 709. (d) For cancerostatic activity of 1,3-selenazoles see:Srivastava, P. C.; Robins, R. K. J. Med. Chem. 1983, 26, 445. (e) Shafiee, A.; Mazloumi, A.; Cohen, V. J. J. Heterocycl. Chem. 1979, 16, 1563.

4. (a) Ueda, S.; Terauchi, H.; Suzuki, K.; Yano, A.; Matsumoto, M.; Kubo, T.; Minato, H.; Arai, Y.; Tsuji, J.; Watanabe, N. Bioorg. Med. Chem. Lett. 2005, 15, 1361. (b) Park, Y.-J.; Koketsu, M.; Kim, J. M.; Yeo, J.-H.; Ishihara, H.; Lee, K.-G.; Kim, S. Y.; Kim, C.-K. Biol. Pharm. Bull. 2003, 26, 1657.

5. Sekhiguchi, A.; Nishina, A.; Kimura, H.; Fukumoto, R.-H.; Koichi, K.; Ishihara, H.; Koketsu, M. Chem. Pharm. Bull. 2005, 53, 1439.

6. (a) Kantlehner, W.; Hauber, M.; Vettel, M. J. Prakt. Chem. 1996, 338, 403. (b) Keil, D.; Hartmann. H. Dyes and Pigments 2000, 44, 149. (c) Keil, D.; Hartmann. H.; Zug, I.; Schroder, A. J Prakt Chem. 2000, 42, 169.

7. (a) Larsen, R. 1,3-Selenazoles, In Comprehensive Heterocyclic Chemistry II, Vol. 3; Shinkai, I.; Katritzky, A.; Rees, C. W.; Scriven, E. F. V., Eds.; Elsevier Science: Oxford, 1996, Chapt.8. (b) Wirth, T. Organoselenium Chemistry. Modern Developments in Organic Synthesis; Springer: Berlin, 2000.

8. (a) Narender, M.; Reddy, M. S.; Pavan kumar, V.; Reddy, V. P.; Nageswar, Y. V. D.; Rao, K. R. J. Org. Chem. 2007, 72, 1849. (b) Koketsu, M.; Kogami, M.; Ando, H.; Ishihara, H. Synthesis 2006, 31. (c) H. Below, H.; Pfeiffer, W.-D.; Geisler, K.; Lalk, M.; Langer, P. Eur. J. Org. chem. 2005, 3637. (d) Geisler, K.; Künzler, A.; Below, H.; Bulka, E.; Pfeiffer, W.-D.; Langer, P. Synthesis 2004, 97. (e) Koketsu, M.; Mio, T.; Ishihara, H. Synthesis 2004, 233. (f) Keil, D.; Hartman, H. Phosphorus, sulfur and silicon 1999, 152, 169. (g) Maeda, H.; Kambe, N.; Sonoda, N.; Fujiwara, S.-I.; Siniike, T. Tetrahedron 1997, 53, 3667. (h) Zingaro, R. A.; Bennett, F. C.; Hammer, Jr. G. W. J. Org. Chem. 1953, 18, 292.

9. Koketsu, M.; Tanahka, H.; Ishihara, H. Chem. Lett. 2005, 34, 1260.

10. (a) Li, C.-J.; Chan, T.-H. Organic Reactions in Aqueous Media, John Wiley \& Sons, New York, 1997. (b) Organic Synthesis in Water, Ed. P. A. Grieco, Blackie Academic and Professional, London, 1998. (c) Demko, Z. P.; Sharpless, K. B. J. Org. Chem. 2001, 66, 7945. (d) Li, C. J. Chem. Rev. 2005, 105, 3095.

11. (a) Welton, T. Chem. Rev. 1999, 99, 2071. (b) Wasserscheid, P.; Keim, W. Angew. Chem., Int. Ed. 2000, 39, 3773. (c) Sheldon, R. Chem. Commun. 2001, 2399. (d) Jain, N.; Kumar, A.; Chauhan, S.; Chauhan, S. M. S. Tetrahedron 2005, 61, 1015. 
12. (a) Zhao, Y.-B.; Yan, Z.-Y.; Liang, Y.-M. Tetrahedron Lett. 2006, 47, 1545. (b) Chen, J.; Su, W.; Wu, H.; Liu, M.; Jin, C. Green Chem. 2007, 9, 972.

13. (a) Palimkar, S. S.; Siddiqui, S. A.; Daniel, T.; Lahoti, R. J.; Srinivasan, K. V. J. Org. Chem. 2003, 68, 9371. (b) Potewar, T. M.; Nadaf, R.; Daniel, T.; Lahoti, R. J.; Srinivasan, K. V. Synth. Commun. 2005, 35, 231. (c) Potewar, T. M.; Daniel, T.; Lahoti, R. J.; Srinivasan, K. V. Synth. Commun. 2007, 37, 261. (d) Potewar, T. M.; Siddiqui, S. A.; Lahoti, R. J.; Srinivasan, K. V. Tetrahedron Lett. 2007, 48, 1721. (e) Potewar, T. M.; Ingale S. A.; Srinivasan, K. V. Tetrahedron 2007, 63, 11066. 